\title{
Dielectric properties analysis of paper capacitor
}

Cite as: AIP Conference Proceedings 2269, 030020 (2020); https://doi.org/10.1063/5.0019831

Published Online: 12 October 2020

G. Sree Lakshmi, Rubanenko Oleksandr, Rubanenko Olena, and Labzun Mikhail
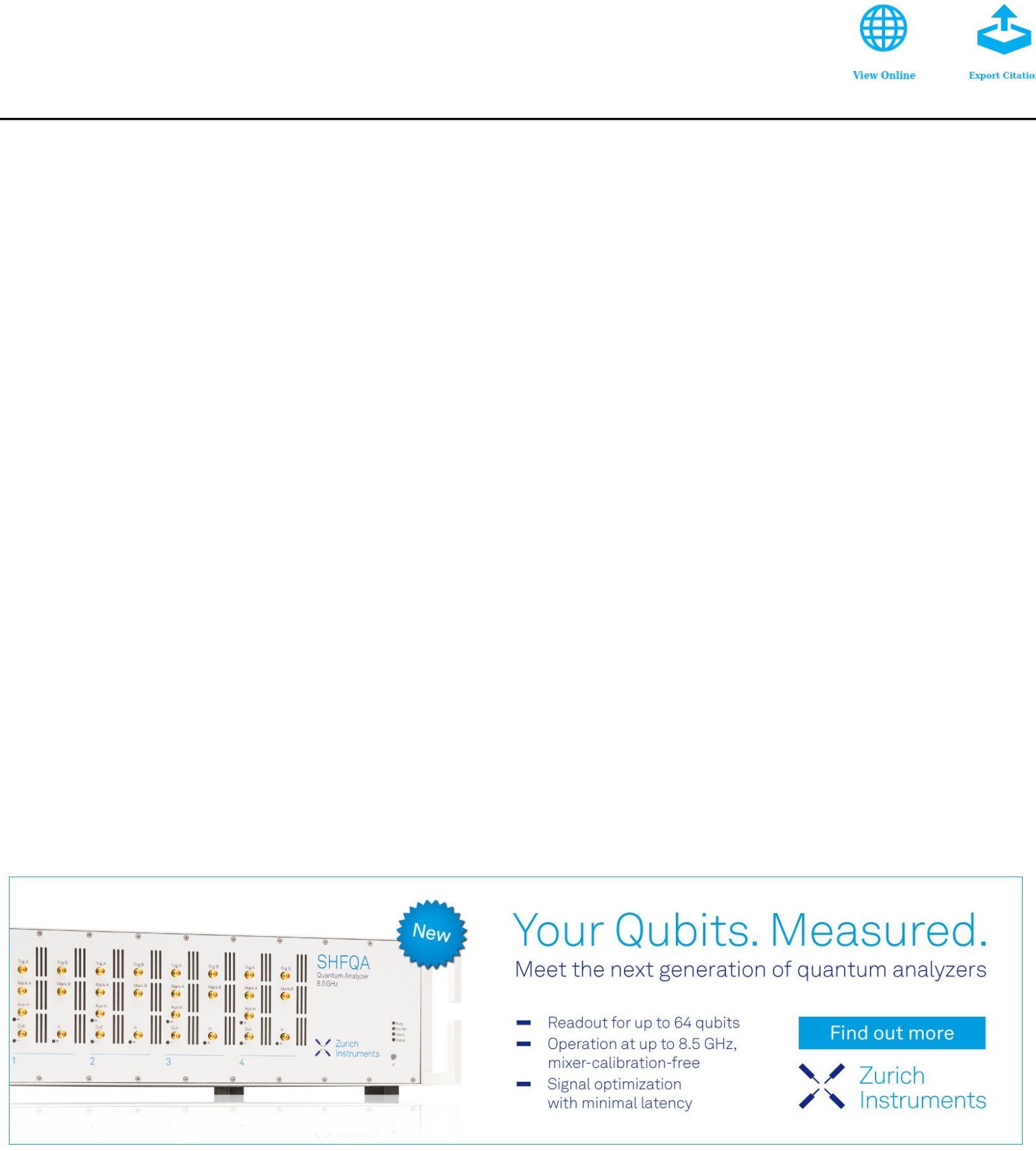


\title{
Dielectric Properties Analysis of Paper Capacitor
}

\author{
G. Sree Lakshmi ${ }^{1 \mathrm{a}}$, Rubanenko Oleksandr $^{2}$, Rubanenko Olena ${ }^{3 b}$ \\ and Labzun Mikhail ${ }^{4}$ \\ ${ }^{1}$ Academic Researcher, Faculty of EE, RICE, UWB, Pilsen, Czech Republic \& Professor, EEE Department,CVR \\ College of Engineering, Hyderabad, India. \\ ${ }^{2}$ Department of Electric Stations and Systems of Vinnytsia National Technical University, 21000, Vinnytsia, Ukraine \\ ${ }^{3}$ Academic Researcher, Faculty of EE, RICE, UWB, Pilsen, Czech Republic. \\ ${ }^{4}$ State EnterpriseNationalenergycompany UKRENERGO, South-Western ES, DepartmentofElectricalSubstations, \\ DivisionofDiagnostics, 21000, Vinnytsia, Ukraine

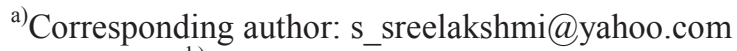 \\ b) olenarubanenko@ukr.net
}

\begin{abstract}
The film-impregnated dielectric under operating conditions is subject to prolonged exposure to electric and thermal fields, as well as mechanical loading. In addition to critical partial discharges, the most serious drawback that impairs the film and condenser reliability in general is the deterioration of the electro-physical characteristics of the polypropylene film and liquid dielectric due to the gradual dissolution of the polymer in the liquid dielectric, which is determined by their chemical composition. This interaction of components, which was investigated in detail, has a thermo activation nature, which relates to the processes of rearrangement of atoms or molecules with the overcoming of potential barriers, and is manifested in the swelling of the polypropylene film with subsequent dissolution of the amorphous component in the polymeric component. As a consequence, irreversible structural changes of the film occur, which lead to a decrease in its electrical and mechanical strength, as well as to an increase in the dielectric loss of the liquid dielectric. Capacitor (Fig. 1 (a)) is designed to provide high-frequency communication at frequencies from 24 to 1500 $\mathrm{kHz}$ in power lines with a nominal voltage of 35,110,150,220,330, $500 \mathrm{kV}$ AC 50 and $60 \mathrm{~Hz}$. Coupling capacitor impregnated with environmentally friendly liquid - condenser oil. The short-circuits consist of capacitor sections (Fig. 1 (b)): a thin layer of dielectric is laid between two thin wide metal strips, and the dielectric film is placed on top and below the metal strips - capacitor covers. This paper gives the detail analysis of dielectric properties of capacitor paper.
\end{abstract}
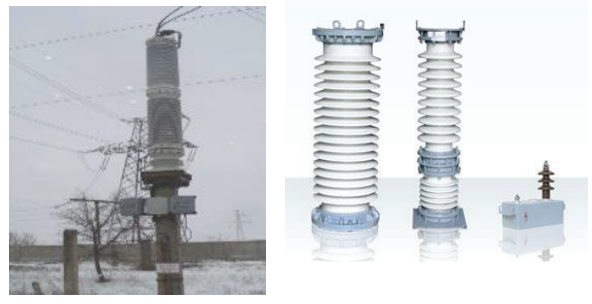

a)
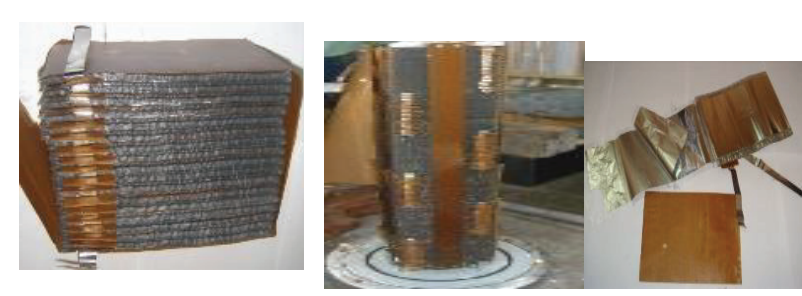

b)

FIGURE 1.a) Capacitor connection; b)capacitor section and condenser paper for extra insulation between sections

\section{INTRODUCTION}

As the networks of high-voltage power transmission lines develop, their length is extended and the automatics are needed, there is a need for reliable dispatching and administrative-economic communication between individual points, the transmission of television signals, emergency switch-off of switches, relay protection of other data on the current mode parameters and electrical equipment. One of the elements involved in this process is communication capacitors, which separate the low-voltage equipment from the high voltage, passing high-frequency signals through

International Conference on Multifunctional Materials (ICMM-2019) AIP Conf. Proc. 2269, 030020-1-030020-6; https://doi.org/10.1063/5.0019831 Published by AIP Publishing. 978-0-7354-2032-8/\$30.00 
the communication channels. These capacitors are also used to connect the communication equipment to the transmission lines.The technological process of generation, transportation, distribution and consumption of electricity, depending on the degree of complexity, responsibility and duration, requires accurate information and quality equipment. This is the reason for the introduction of the latest technologies in the power industry, which in turn require quality operation and maintenance, as the cost of high-voltage equipment is a significant indicator.

Safe and high-quality operation of high-voltage equipment is possible only with the use of modern reliable and high-quality electrical materials. However, accidents at the enterprises of the electric power industry caused by damage to new types of high-voltage equipment indicate the need for qualitative studies of the causes of these damages, studies of the processes that occur in insulating materials during their operation.Such research is needed not only for the creation of new insulation materials but also for the improvement of technologies for the manufacture of responsible, high-value equipment, structures, and methods of operation[1-5].

High-voltage coupling capacitors operated on $330 \mathrm{kV}$ overhead lines in Ukraine, Russia and many other countries are no exception. The research conducted by the authors, the results of which are presented in this article, are caused by damages of capacitors of coupling type CMA-166, produced by the Ust-Kamenogorsk Condenser Plant (Kazakhstan), which took place in Ukraine.Damage to the operating circuit causes not only the power lines to be switched off, but also poses a threat to the safety of the substation personnel. Therefore, conducted research is relevant. To rationalize the decommissioning of the CMA-166 coupling capacitors with degraded properties of polymer insulation (isopropylene film).In accordance with the purpose in the article the results of the following tasks are presented:

1.Research of design features caused by replacement of capacitor-type paper-oil insulation by polymeric insulation in coupling capacitors.

2.Analysis of capacitor coupling damage.

3.Using the neuro-fuzzy simulation method to determine the residual life of the CMA-166 capacitor during operation.

4.Research of the method of periodic monitoring of the condition of the capacitors in the operating conditions.

\section{RESEARCH RESULTS}

Insulation is one of the important elements in the design of high voltage capacitors. It largely determines the size and reliability of the work. Various insulating materials are used to insulate the conductive parts of the AC.Since the isolation of 1-750 kV high-voltage equipment is one of the most widespread and responsible elements of electrical equipment of power systems, special control systems have been created and developed to evaluate its technical condition [6]. They control equipment and increase reliability at power plants and large sub-stations.However, there is damage to the insulation material of the equipment which has not fulfilled its passport resource. Among such equipment are high-voltage capacitors. The traditional insulating material in such capacitors is olive paper insulation of the capacitor type. However, in modern capacitors, this insulating material is replaced by polypropylene. Unfortunately, high voltage capacitors with polypropylene insulation are damaged during operation.

The communication capacitors are designed to provide high frequency communication at frequencies of 24 $1500 \mathrm{kHz}$ in power lines with a nominal voltage of $35-750 \mathrm{kV}$. On the basis of high-voltage capacitors, measuring devices (voltage dividers, non-resonant voltage transformers, etc.) are made, as well as power take-off devices for supplying measuring equipment and power equipment. Due to the importance of capacitors that are solved using coupling capacitors (CC), their reliability indicators are subject to high requirements. So, for example, the average life of the short circuit type SMA-166 / $\sqrt{3}-14$ UHL1, according to the passport data, is 25 years, and the probability of failure-free operation - 0.9.The basic assessment of the insulation status of the $330 \mathrm{kV}$ short circuit is made during periodic inspections with the equipment switched off. The investigated capacitor SMA-166 / -143-14 UHL1 is designed to provide high-frequency communication at frequencies from 24 to $1500 \mathrm{kHz}$ in power lines with a nominal voltage of 35, 110, 150, 220,330, $500 \mathrm{kV}$ AC 50 and $60 \mathrm{~Hz}$. CMA-166 / 3-14 UHL1 impregnated with environmentally friendly liquid and condenser oil. The life of the capacitor SMA-166 / v3-14 UHL1 is at least 25 years.

a) Design features and materials for making capacitors.

The CC consist of capacitor sections (Fig. 1 (a)): a thin layer of dielectric is laid between two thin wide metal strips, in addition, the dielectric film is placed on top and below the metal strips - capacitor covers. To prevent the capacitor covers from closing at the edges of the section, the dielectric film is made $20 \mathrm{~mm}$ wider than the covers. Each metal strip is output and the capacitor thus obtained is twisted into a flat section [2].The material for the 
capacitor covers is an aluminum foil with a thickness of 5-8 microns. As a dielectric between the covers of a section in modern short-circuits, a polypropylene film with a thickness of 4-7 $\mu \mathrm{m}$ of isotactic polypropylene (or thickness of one sheet of $10 \mu \mathrm{m}$, total dielectric $40 \mu \mathrm{m}$, foil $10 \mu \mathrm{m}$ ) is used, which provides the best performance of the capacitor in alternating and pulsed modes, compared to condenser paper 10-15 microns thick used in "old" capacitors and other organic films; the thickness of one sheet is $10 \mu \mathrm{m}$, the total dielectric is $40 \mu \mathrm{m}$, the foil is $10 \mu \mathrm{m}$. Isotactic polypropylene is produced by the polymerization of propylene $\mathrm{CH}_{2}-\mathrm{CH} \cdot \mathrm{CH}_{3}$, which results in the formation of an isotactic structure under certain conditions, characterized by the location of the $\mathrm{CH}_{3}$ radical on one side of the main chain [1-5].

The capacitor sections are connected by pins to each other (Fig.1 (b)) and the packages into groups. The sections additionally insulate with each other a layer of capacitor paper as well as the packages. The bundled sections are bonded together as shown in Fig.1 (b). Modern CC has only one bundle of bundles in series, using non-soldering technology to provide electrical communication between the bundles. The bonded packages are mounted inside a porcelain body that is cylindrical in shape.The top of the tire is fitted with an expander in the form of membrane boxes [6] (bellows) to compensate for the temperature fluctuations of the environment.During the production of capacitors, the film dielectric and the condenser paper are heat-vacuum treated.To eliminate the air that is between the layers of solid materials and in the pores of the paper, sections and packages are impregnated with a special liquid dielectric that has high electrical strength and electrical permeability. As a liquid for condenser seepage, which is intended for use on alternating voltage, the most commonly used purified condenser oil or phenyl xylethane. The aromatic structure of the hydrocarbon dielectric fluid provides increased gas resistance and stability of polypropylene insulation [6]. Drying and degassing of the liquid dielectric, which is poured into the capacitor, is carried out at a temperature of $80^{\circ} \mathrm{C}$ and the final pressure is not lower than the saturated vapor pressure of the liquid at the drying temperature.

Porcelain insulator (body), which has a developed lateral surface (ribs), to increase the length of the surface path of leakage, sealed on both sides by metal flanges.

Rubber seals are used to tighten the coupling element.

b) Basic parameters of capacitors of connection

The main parameters of the $\mathrm{CC}$ are [7-9]:

- $\quad$ loss tangent $(\operatorname{tg} \delta)$;

- $\quad$ electrical capacitor insulation resistance - DC electrical resistance of the capacitor, which is determined by the ratio $R_{\mathrm{iz}}=\mathrm{U} / \mathrm{I}_{\text {turn }}$, where $\mathrm{U}$ is the voltage applied to the capacitor, $\mathrm{I}_{\text {turn }}$ is the leakage current or conductivity;

- the time constant, which is measured in seconds and equals the product of the insulation resistance to the value of the rated capacity;

- $\quad$ the insulation resistance between the housing and the connected terminals of the capacitor;

- $\quad$ frequency properties;

- the nature of the dependence of the capacitor capacitance (taking into account the influence of the parameters $L_{c}$ and $R_{n}$ ) in the frequency range from zero to $f_{p}$ is determined by the ratio of parameters $C$, $\mathrm{LC}, \mathrm{R}_{\mathrm{n}}$;

- $\quad$ the allowable amplitude of the alternating voltage on the capacitor $\mathrm{U}_{\mathrm{m} . \mathrm{add}}$;

The temperature coefficient of capacitance (TCC) - a parameter used to characterize capacitors by the linear dependence of capacitance on temperature.

\section{FAULTS STATISTICS OF CC}

Processes that occur in capacitors under the conditions of ionizing radiation lead to reversible and irreversible changes in electrical parameters. Reversible changes are related to the processes of ionization of dielectric materials and air and are accompanied by a sharp decrease in insulation resistance and an increase in leakage current. The loss angle tangent is also increasing, especially at low frequencies. Upon termination of the irradiation, the insulation resistance (leakage current of the oxide capacitors) in most cases is restored. Residual changes in parameters are mainly related to persistent violations of the structure of the working dielectric and protective materials. The structure of polymeric materials used in film and combined capacitors is particularly prone to change.

Capacitors with organic dielectrics are generally more sensitive to the effects of radiation than inorganic dielectrics. The most resistant to ionizing radiation are ceramic capacitors. The largest irreversible changes in parameters are caused by prolonged exposure to an electrical load.Exceeding the permissible values of DC and AC 
sharply reduces the reliability of the capacitors.At the same time, in 2013, 12 elements (out of 30 installed) of shortcircuits for $330 \mathrm{kV}$ class of type CMA-166 / -3-14 UHL, 2008 of production, production of LLC "UKKZ" (UstKamenogorsk Condenser Plant, were detected in the power system), Kazakhstan, which have poor insulation performance over a lifetime of 5 years. The sharp deterioration of the insulation parameters of such a number of short circuits ( $40 \%$ of elements) requires increased attention not only to the "old" but also to the "new" capacitors of this technological batch.

Damage to the operating circuit causes not only the power lines to be switched off, but also poses a threat to the safety of the substation personnel.During the period 2002-2010, 11 technological disturbances in the operation of high-voltage capacitors of connection (CC) with a lifetime of 24-36 years occurred at the substations of SE "Ukrenergo" (Fig. 2).Processes that occur in capacitors under the conditions of ionizing radiation lead to reversible and irreversible changes in electrical parameters.Reversible changes are related to the processes of ionization of dielectric materials and air and are accompanied by a sharp decrease in insulation resistance and an increase in leakage current. The loss angle tangent is also increasing, especially at low frequencies. Upon termination of the irradiation, the insulation resistance (leakage current of the oxide capacitors) in most cases is restored. Residual changes in parameters are mainly related to persistent violations of the structure of the working dielectric and protective materials. The structure of polymeric materials used in film and combined capacitors is particularly prone to change.Capacitors with organic dielectrics are generally more sensitive to the effects of radiation than inorganic dielectrics. The most resistant to ionizing radiation are ceramic capacitors.

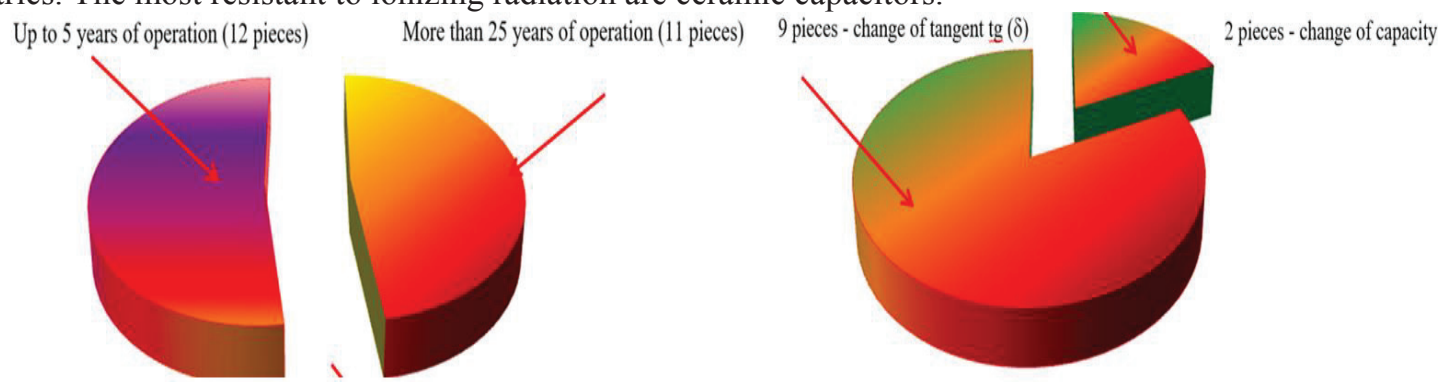

FIGURE 2.Deterioration of the characteristics of the CC

For the period 2008-2013, 11 elements of the capacitors of 110-330 kV voltage classes, which have been operated for more than 25 years, with isolation characteristics that do not meet the requirements of the test norms have been detected in the Southwestern EU [6]: by the tangent of dielectric loss angle - 9 pcs., for changing the capacity -2 pcs.

Characteristic defects of "old" capacitors. Consider the main defects of the "old" capacitors and change the diagnostic parameters.

1. The general gradual aging of the condenser paper or the oxidation or wetting of the condenser oil: a general increase in the temperature of the tire, a significant increase in tg $\delta$.

2. Terminal break that provides electrical connection between one of the flanges and the capacitor group or between packages: no excess of temperature, capacity and tg $\delta$ are not measured correctly - very small values.

3. Partial discharges or breakdown of part of sections: increase of capacity value, local heating of a tire in defective zones.

4. The moisture of the stand insulator: reduction of the insulator resistance.

According to the statistics of rejection of "old" CC in the Southwestern EU over the last 5 years, the main reason for the deterioration of insulation is its aging, which is accompanied by an overall increase in tg $\delta$. On this basis, there are 9 capacitor elements out of 11 discarded during this period.The 2 elements found an overtime increase in capacity due to the electrical overlap of part of the packages. For the "old" capacitors, the characteristic reason for the increase in capacitance is the overlap on the end surface of the packages, with traces of surface arc discharges, due to the aging of the insulation and the considerable unevenness of the electric field near the edges of the covers.Investigation of the causes of deterioration of the insulation of "new" capacitors due to disassembly of the defective element. Due to a significant change in the capacitance and the nature of the temperature profile on the condenser height, it was suggested that full or partial breakdown of part of the condenser packages. The results of measuring the capacitance packet values are shown in table 1 . 
TABLE1. Results of measurements of capacitance packet values of the element

\begin{tabular}{ccccccc}
\hline №,Package & 1 & 2 & 3 & 4 & 5 & 6 \\
\hline Capacity, mF & 220 & 564 & 184 & 112 & 89 & 79 \\
\hline
\end{tabular}

The capacitor element has been parsed to verify this assumption.During a detailed visual examination, no clusters, electrical overlaps, arc discharges or other defects were found in the group and the packages (Fig. 2) attached, as well as during the inspection of the membrane boxes. There are no comments on liquid dielectric on the results of physicochemical analyzes. The estimated capacity of the package is $82.8 \mathrm{nF}$, according to the measurement results, the capacities of the $5^{\text {th }}$ and $6^{\text {th }}$ packages correspond to the calculated values. The varying degree of damage to the packets is explained by the uneven distribution of voltage across the packets within the element.

The insulation resistance of the individual packages was more than 50,000 M $\Omega$, which indicates a significant supply of their electrical strength in the absence of defects, however, when measuring the isolation of the individual capacitor sections of the upper packages with a constant voltage of $1 \mathrm{kV}$, part of the sections was completely shortened, and the sections were less $M \Omega$. A significant increase in capacity and a reduction in the insulation of individual sections indicate a partial breakdown of the packages.Inspection of the individual condenser sections during their unwinding revealed deformation of the dielectric film and foil in the form of folds in almost all sections (Fig. 3 (a), (b), (c), (d)) which is classified as a factory defect related to the technology of section winding.In some sections from the places of deformation of the foil and the film in the inner layers of the section the traces of electrical breakdown are clearly traced, which are quite often accompanied by traces of decomposition of the organic film and liquid dielectric (Fig. 3 (a)). The breakdown marks in the inner layers of individual sections are poorly expressed, and in some sections they pass through all the layers and go to their outer side (Fig. 3 (b)).In some sections, there are significant deformations in the inner layers.

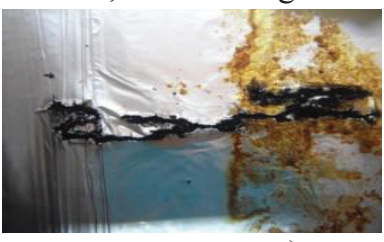

a)

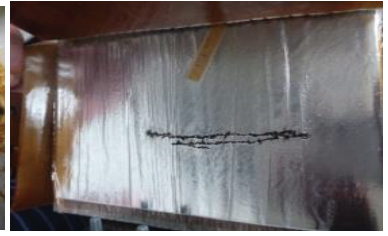

b)

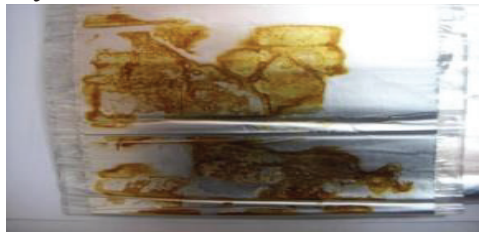

c)

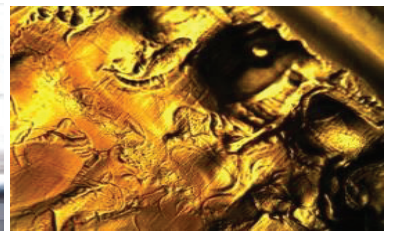

d)

FIGURE 3.Traces of the breakdown of the section with deformation of the foil and dielectric film and traces of decomposition of the dielectric film and liquid dielectric (a); traces of electrical breakdown of the section, which begin at places of deformation of the organic film and reach the surface of the section (b); Traces of decomposition of organic film and liquid dielectric without visible breakdown (c); and Traces of the beginning of the breakdown of a dielectric film, with traces of its decomposition in a liquid dielectric, at the point of its deformation, detected under a microscope (d).

Fig.4 (a) for comparison shows a photograph under the microscope of a fragment of a dielectric film, in which no signs of defects were detected. In some cases, a point breakdown of the film was detected in the sections without visible deformation (Fig.4 (b)).Photograph under film microscope without signs of defects. In some cases, a point breakdown of the film was detected in the sections without visible deformation (Fig.4 (c)).

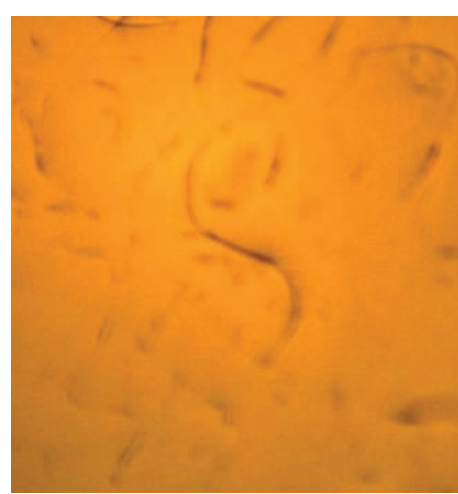

a)

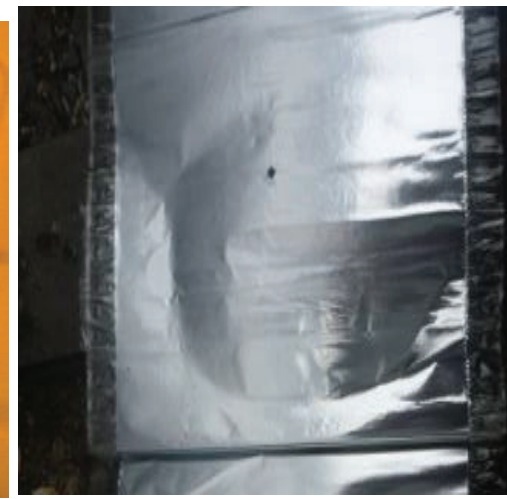

b)

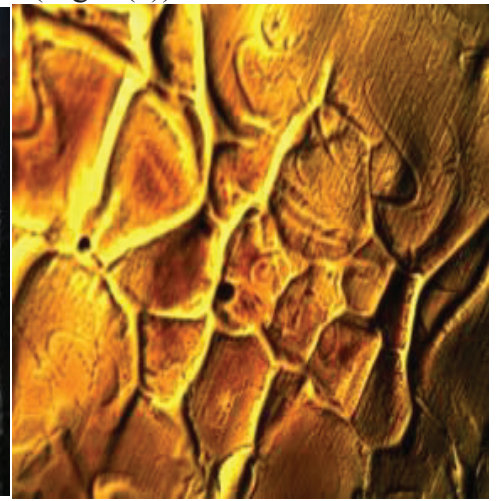

c)

FIGURE 4. Photograph under film microscope without signs of defects(a); Point breakdown of the section where there is no significant deformation of the dielectric film (b) and Point breakdown of a dielectric film with traces of its decomposition in a liquid dielectric (c). 
The film-impregnated dielectric under operating conditions is subject to prolonged exposure to electric and thermal fields, as well as mechanical loading. In addition to critical partial discharges, the most serious drawback that impairs the reliability of the film and the capacitor, in general, is the deterioration of the electrophysical characteristics of the polypropylene film and aromatic liquid dielectric due to the gradual dissolution of the polymer in the liquid dielectric, which is determined by their chemical composition.This interaction of the components, which was thoroughly investigated, has a thermoactivation nature, that is, relates to the processes of rearrangement of atoms or molecules with the overcoming of potential barriers, and is manifested in the swelling of the polypropylene film, followed by dissolution of the amorphous polymer component in the liquid. As a consequence, irreversible structural changes in the film result in a decrease in its electrical and mechanical strength, as well as an increase in the dielectric loss of the liquid dielectric. Traces of decomposition of the dielectric film and liquid dielectric in the inner layers of sections without visible deformation were detected, with the trace of the breakdown detected only under the microscope (Fig. 9). The presence of the last two defects suggests that the cause of the breakdown of the condenser sections, in addition to deformation of the film and foil, may also be a mismatch of the dielectric properties of the organic film to the requirements put forward by it in the production of capacitors, to satisfy the requirement to ensure the probability of working for up to 20 years not less than 0.9. Note that the capacitors of this process batch are characterized by the breakdown of the inner layers of the capacitor sections and packages, as opposed to the electrical breakdown of the "old" capacitors. Breakdown of the sections can lead to the loss of capacitor isolation as a whole. Damage to the capacitors is almost always accompanied by an explosion and a danger to the personnel of the facility.

\section{CONCLUSION}

1. $40 \%$ of batch of capacitors of connection for a voltage class of $330 \mathrm{kV}$ type CMA-166 / $\sqrt{3}-14$ УХЛ1, 2008 in., "UKKZ", have unsatisfactory isolation characteristics at the term of operation - 5 years, and their indicators of reliability do not meet the requirements of GOST and passport data.

2. The cause of the deterioration of the insulation characteristics of the capacitors is the factory defects: deformation of the polypropylene film due to the violation of the technology of winding of the capacitor sections, and the mismatch of the chemical resistance of the dielectric film to dissolution in the liquid dielectric.

3. The main diagnostic parameter that characterizes the deterioration of capacitor insulation of a given batch is the increase in capacity caused by the partial breakdown of the capacitor sections and packages.

\section{REFERENCES}

1. L. Cheng et al., "Polypropylene nanocomposite for power equipment: a review," in IET Nanodielectrics, vol. 1, no. 2, pp. 92-103, 7 2018. doi: 10.1049/iet-nde.2018.000

2. M. Ritamäki, I. Rytöluoto and K. Lahti, "Performance metrics for a modern BOPP capacitor film," in IEEE Transactions on Dielectrics and Electrical Insulation, vol. 26, no. 4, pp. 1229-1237, Aug. 2019. doi: 10.1109/TDEI.2019.007970

3. C. Zhang, S. Xie and Y. Zhang, "Repeated impulse aging of oil-polypropylene insulation," in IEEE Transactions on Dielectrics and Electrical Insulation, vol. 26, no. 5, pp. 1581-1587, Oct. 2019. doi: 10.1109/TDEI.2019.008180

4. Z. Chen et al., "Experimental Study of the Breakdown Characteristics of Polypropylene Films Under Nanosecond Voltage Pulses," in IEEE Transactions on Plasma Science, vol. 46, no. 11, pp. 4010-4016, Nov. 2018. doi: 10.1109/TPS.2018.2866061

5. H. Li et al., "Mechanism of insulation resistance in metalized polypropylene film capacitors," 2013 Annual Report Conference on Electrical Insulation and Dielectric Phenomena, Shenzhen, 2013, pp. 643-646. doi: 10.1109/CEIDP.2013.6748258

6. Parametric failures of CMA -166 / 3capacitor in the South-West electric power system of Ukraine M.P. Labzun, P.D. Lezhnyuk, A.E. Rubanenko, V.V. Rudakov, 2014NTU "KhPI". 2014. No 21 (1064)

7. Norms of vibrating electro-cooling: SOU-N EE 20.302: 2007.: - K.: GRIFRE, 2007.-262 p. - (Normative document of Ministry of Energy of Ukraine).

8. Communication and power take-off capacitors for power lines: GOST 15581-80 - [Date of introduction 198101-07]. - M.: IPK Publishing House of Standards, 1989. - 41 p.

Zvenigorodsky I.S. Communication and power take-off capacitors. - M.: Energy, 1969. - 64 p. 